\title{
Life Contexts Make a Difference: Emotional Stability in Younger and Older Adults
}

\author{
Annette Brose \\ Max Planck Institute for Human Development
}

\author{
Susanne Scheibe \\ University of Groningen
}

\author{
Florian Schmiedek \\ Max Planck Institute for Human Development and German Institute for International Educational Research (DIPF)
}

\begin{abstract}
Emotional stability, as indicated by low affect variability and low affective reactivity to daily events, for example, tends to increase across the adult life span. This study investigated a contextual explanation for such age differences, relating affect variability and affective reactivity to age-groupspecific life contexts. A sample of 101 younger and 103 older adults reported daily stressors and negative affect across 100 days. Compared with younger adults, older adults (a) experienced fewer stressors overall, (b) had less heterogeneous stressor profiles, and (c) reported that stressors had less impact on daily routines. As expected, these contextual factors were relevant for interindividual differences in emotional stability. Multiple regression analyses revealed that reduced affect variability and affective reactivity in older adults were associated with these age-group specific life contexts. Moreover, matching younger and older adults on the contextual factors to explore the effects of context on age-group differences further provided support for the (partially) contextual explanation of age differences in emotional stability. Matched subgroups of younger and older adults that were comparable on contextual variables were identified. Affective variability, but not affective reactivity, was more similar in the matched subsamples than in the total samples of younger and older adults. We conclude that contexts in which affective experiences emerge require more attention when aiming to explain interindividual and age group differences in emotional stability. Moreover, future studies need to disentangle the extent to which contexts interact with active self-regulatory processes to shape affective experiences across adulthood.
\end{abstract}

Keywords: affect variability, affective reactivity, context, emotional development, aging, propensity score matching

Mastery of life's inevitable ups and downs requires emotionregulatory skills. Accordingly, improved emotion regulation skills

This article was published Online First October 15, 2012.

Annette Brose, Center for Lifespan Psychology, Max Planck Institute for Human Development, Berlin, Germany; Susanne Scheibe, Department of Organizational Psychology, University of Groningen, Groningen, The Netherlands; Florian Schmiedek, Center for Lifespan Psychology, Max Planck Institute for Human Development, and German Institute for International Educational Research (DIPF), Frankfurt/Main, Germany.

We thank Martin Lövdén, Colin Bauer, Annette Rentz-Lühning, and Julia Wolff for their important roles in conducting the COGITO Study. We also thank Ulman Lindenberger and Michael Becker for valuable discussions on propensity score matching and Julia Delius for improving the English. The COGITO Study was supported by the Max Planck Society, including a grant from the innovation fund of the Max Planck Society (M.FE.A .BILD0005); the Sofja Kovalevskaja Award (to Martin Lövdén) of the Alexander von Humboldt Foundation donated by the German Federal Ministry for Education and Research (BMBF); the German Research Foundation (DFG; KFG 163); and the BMBF (CAI).

Correspondence concerning this article should be addressed to Annette Brose, Center for Lifespan Psychology, Max Planck Institute for Human Development, Lentzeallee 94, 14195 Berlin, Germany. E-mail: brose@ mpib-berlin.mpg.de with age are a prominent explanation for the finding that older adults show comparatively high levels of well-being in comparison with younger adults (Carstensen, Isaacowitz, \& Charles, 1999; Scheibe \& Carstensen, 2010). But is this perspective giving us the whole picture? In this study, we suggest that emotional outcomes are also shaped by life contexts that change across adulthood. The better the circumstances are that surround emotionally taxing events, the easier it should be to regulate emotions and the fewer skills should be required. This contextual perspective is not reflected well in the literature on emotional development. Systematic research investigating whether adult age differences in emotional functioning are related to changing characteristics of the environment is sparse (but see Charles et al., 2010). Yet, it seems plausible that a more tranquil lifestyle and less stressful daily lives may contribute to older adults' emotional stability and well-being. For example, being confronted with a stressor may be particularly taxing if a person is confronted with multiple stressors simultaneously or if she or he is generally overburdened (van Eck, Nicolson, \& Berkhof, 1998). This scenario seems to resemble younger adults' lives more than older adults' (Stawski, Sliwinski, Almeida, \& Smyth, 2008). We therefore investigated whether differences in life contexts (i.e., in stressor frequency, stressor heterogeneity, and stressor impact on daily routines) contribute to adult age differences in two aspects of emotional 
stability, namely affect variability and affective reactivity to daily stressors.

\section{Age Differences in Emotional Stability}

To understand emotional well-being across adulthood, it is not sufficient to consider mean levels of affect. Emotional well-being has multiple dynamic components, including affect variability and affective reactivity to stressful events. Affect variability is the fluctuation of affective states around relatively stable levels of affect attributable to various internal and external influences as well as the interaction of the individual with the environment (Eid \& Diener, 1999; Nesselroade, 1991; Ram \& Gerstorf, 2009). Affect variability is considered a trait; that is, a relatively stable interindividual differences characteristic. External and internal influences on affect variability include, for example, the weather, circadian rhythms, physical activity, and stressors (Kuppens, Oravecz, \& Tuerlinckx, 2010). These influences are complexly intertwined and can be either conscious or unconscious (e.g., when being cold influences state affect judgments without the person noticing; Koole, 2009; Robinson \& Clore, 2002;). Affective reactivity describes a change in affect that occurs in temporal proximity to a threatening event (i.e., a stressor) and interacts with appraisals and actions, reflecting situation-specific coping (Lazarus \& Folkman, 1984). In this sense affective reactivity represents one of many possible causes of affect variability.

Both affect variability and reactivity reflect dynamic aspects of an individual's emotional functioning, pointing to processes of self-regulation (Carver \& Scheier, 1999). Both also exhibit interindividual differences (i.e., some individuals are more variable and/or reactive than others) and have been linked to neuroticism (i.e., dispositional affective responsiveness; Bolger \& Zuckerman, 1995; Eid \& Diener, 1999; Eysenck, 1990; Williams, 1990). Given the latter commonality, those individuals with greater affective variability should also react more strongly when specific events occur. Moreover, individuals who experience stressors more frequently should also be more variable.

At the same time, affect variability and reactivity are distinguishable. Although stressors are but one of many causes of affect variability, they are the defining cause of affective reactivity. An up and down reflected in variability may partly go unnoticed and may not elicit explicit actions to return to equilibrium, whereas reactivity in circumscribed situations signals a deviation from desired states that requires action. In addition, high variability does not necessarily imply high reactivity, for example, if an individual is particularly skilled in handling stressful events.

The aging literature suggests that affect variability is reduced in older ages. Self-report studies (Lawton, Kleban, Rajagopal, \& Dean, 1992) converge with experience-sampling and laboratory studies that sampled affective experiences across hours or days in showing reduced affect variability with increasing age (Carstensen, Mayr, Pasupathi, \& Nesselroade, 2000; Röcke, Li, \& Smith, 2009). Affective reactivity to daily stressors also appears to decrease with age, both on shorter and longer time scales such as hours and days (Brose, Schmiedek, Lövdén, \& Lindenberger, 2011; Stawski, Almeida, Lachman, Tun, \& Rosnick, 2010; Uchino, Berg, Smith, Pearce, \& Skinner, 2006). Importantly, the picture here is somewhat diverse and not well integrated, with single studies reporting increased reactivity with age or no age- group differences (Mroczek \& Almeida, 2004; Wrzus, Müller, Wagner, Lindenberger, \& Riediger, 2012).

A number of potential mechanisms have been discussed regarding these age differences in affect variability (for review, see Röcke et al., 2009) and affective reactivity (e.g., Brose et al., 2011). Repeated exposure to emotionally charged situations across the lifetime may lead to increased expertise in dealing with them (Charles, 2010). Specifically, people are thought to gain practice in regulating their emotions to the extent that life events are experienced and resolved across life (Blanchard-Fields, Mienaltowski, \& Seay, 2007; see also Seery, 2011). Moreover, older age has been linked to changes in the motivation to regulate emotions (Carstensen et al., 1999). As time horizons grow shorter, people are thought to shift their priorities from gaining resources that pay off in the future toward improving emotional experience in the presence. Age differences in emotion regulation potentially reflect this shift in priorities. In particular, older adults are thought to use antecedent-focused emotion regulation strategies such as avoidant coping and reappraisal more often than younger adults (John \& Gross, 2004; Shiota \& Levenson, 2009). This suspends emotional episodes early before the emotional reaction gains full force (Gross, 1998). Thus, if applied, such strategies should lead to reduced affect variability and affective reactivity to stressors.

Additionally, habituation may occur so that the intensity of emotional reactions to events is reduced (Frederick \& Loewenstein, 1999). A number of theoretical considerations and findings support the idea that emotional development is partly being driven by adaptation, while others do not (for review, see Luhmann \& Eid, 2009). In sum, improved emotion regulation, higher motivation to regulate emotions, and reduced emotional reactivity as a result of habituation may explain increased emotional stability in older adults. These three mechanisms reside within the individual

\section{Age Differences in Life Contexts}

In addition to the above mechanisms, contextual factors that change as people age may be relevant for emotional stability. Social roles normatively differ between younger and older adults and this shapes the structure of daily lives. ${ }^{1}$ Younger adults typically engage in succeeding at work, establishing intimate relationships, a social network, and family (Erikson, 1968; Havighurst, 1973). In comparison, family related duties are usually reduced in older adulthood and social ties are typically well-established (Kahn \& Antonucci, 1980). Probably the most incisive difference between structural characteristics of younger and older adults' lives is the discontinuation of employment after retirement. Given these shifts in social roles and the less externally determined duties, older adults may engage in fewer life domains than younger adults. They should also have more time to spend at home and to engage in leisure activities, or, more generally, to flexibly engage in activities that are in line with their preferences and thereby less stressful (Freund, Nikitin, \& Ritter, 2009). A factor that can limit daily activities in later life is more fragile functional health.

\footnotetext{
${ }^{1}$ We focus on normative age-group differences in this study and do not elaborate on cohort effects or the idiosyncratic nature of development (Baltes, Lindenberger, \& Staudinger, 2006). We acknowledge that large interindividual differences exist in how daily lives are structured, within and across age groups.
} 
Chronic conditions reduce mobility in older adulthood (i.e., the ability to walk half a mile; Guralnik et al., 1993), and mobility is an essential requirement for engaging in activities outside the home. Together, changes in social roles, duties, and health status point to a more tranquil, less stressful, and less heterogeneous life context in older ages. A more tranquil life context may be further strengthened by older adults' increased preferences for routines (Bouisson, 2002; Kastenbaum, 1981).

Empirical findings are in line with this notion. Older adults spend much time in the immediate home environment (Oswald \& Wahl, 2005), and increasing age is positively associated with time spent on sedentary activities (e.g., watching TV; Rosenkoetter, Garris, \& Engdahl, 2001). Their goals appear more similar regarding the life domains they address than younger adults' (Riediger \& Freund, 2006). Older adults report fewer daily stressors than younger adults (Almeida \& Horn, 2004; see also Zautra, Finch, Reich, \& Guarnaccia, 1991) and their routines are less disrupted by stressors (Almeida \& Horn, 2004), which seems plausible given greater time resources and, accordingly, more flexibility regarding time use.

The first aim of this study was to substantiate these findings on age-differential life contexts with a particular focus on daily stressors over a time span of 100 days. Because of the large number of time points, the current study provides an unprecedented opportunity for investigating age-group differences in daily life contexts, including infrequent and unusual stressors and both relatively usual as well as unusual weeks of people's daily lives. We predicted a lower frequency of daily stressors in older as compared with younger adults, reduced heterogeneity of stressors, more chronic health-related stressors, and a reduced impact of stressors on routines.

\section{Life Context Effects on Emotional Stability}

The changes in life contexts just described are an additional potential cause for age-related differences in affective variability and reactivity (Röcke et al., 2009). Affect variability occurs partly because of the person's interaction with the environment. As Fleeson and Jolly (2006; p. 52) noted, "because variability is defined as state changes across occasions, characteristics of the occasions (e.g., situations) are directly implicated in the concept of within-person variability." Two context characteristics appear particularly relevant for affect variability: the frequency at which stressful events occur and the heterogeneity of stressors (i.e., the distribution of stressors across life domains). The smaller the number of events and the more homogeneous they are, the lower affect variability should be. As described above, just these two characteristics tend to differ in younger and older adults' daily life, suggesting that context characteristics may partly account for affect variability and age-group differences therein. Additionally, stressor impact on daily routines may influence affect variability because it interrupts a more smooth ebb and flow of states across situations. This aspect also seems to vary by age (i.e., routines should be more fragile in younger adults because of more duties and time constraints), which bolsters the expectation that changes in life contexts could be related to the previously observed age differences in affective variability.

Life contexts should also impact on affective reactivity to daily stressors. The strength of stress reactivity is moderated by traits
(Bolger \& Zuckerman, 1995) but also by situational characteristics. The presence of chronic stressors and high levels of perceived stress increases reactivity to stress (Serido, Almeida, \& Wethington, 2004; Sliwinski, Almeida, Smyth, \& Stawski, 2009). Moreover, reactivity varies by stressor type (Bolger et al., 1989), and stressor severity varies across situations within specific stressor types (also discussed as intraevent or intracategory variability; Almeida, Stawski, \& Cichy, 2010; Dohrenwend, 2006). In this study, we argue that three additional contextual characteristics matter for affective reactivity: the frequency of stressor occurrence, the heterogeneity of stressor profiles, and the extent to which stressors disrupt routines. First, if persons experience fewer stressors overall, they should have more resources available to cope with a single stressor when it does occur. Second, if stressors are more homogenous (i.e., the same or very similar stressors reoccur over time), they should be more predictable and it should be easier to establish adequate coping strategies, resulting in reduced reactivity (for a similar argument regarding the role of practice for emotion regulation, see Charles, 2010, and Seery, 2011). Third, if stressors generally have a lower impact on routines, the affective reaction to stressors should be smaller (Lazarus \& Folkman, 1984). Thus, the three stressor characteristics that should differ across age groups should also moderate affective reactivity.

In sum, the second aim of this study was to investigate whether age-group differences in affect variability and affective reactivity are partly related to age-group specific life contexts. Such a finding would be in line with a contextual explanation for increased emotional stability in older adulthood.

\section{Summary of Research Questions and Methodological Approach}

Our research group previously found reduced affect variability and reactivity in older as compared with younger adults (Brose et al., 2011; Wolff, 2007) using the same data as presented in this study. Here, we aim at further informing the previously published results by exploring a potential explanation for the identified age-group differences. Specifically, we expected older adults' lives to be more homogenous and less stressful, as indicated by lower frequency of stressors, lower heterogeneity of stressors, and less impact of stressors on daily routines. Moreover, we expected that these stressor characteristics are associated with reduced affective variability and affective reactivity. Third, we hypothesized that, after adjusting for age differences in stressor characteristics, age differences in affect variability and affect reactivity would be reduced.

When examining the relationship between age and context variables in predicting the two facets of emotional stability, we primarily relied on a matching procedure as the analytical approach. Younger and older adults were matched on context variables (i.e., subgroups of younger and older adults were identified with comparable context variables) to determine a potential impact of life context on age-related differences in emotional stability. Finding more similarity in emotional stability for these matched samples than in the total sample would be in line with our expectation that life context matters for age-group differences in emotional stability. 
Matching was achieved by means of logistic regression analysis as is common in propensity score matching (PSM) ${ }^{2}$ (e.g., Foster, 2010). Recently, matching has been proposed as a nonparametric preprocessing method that is suitable for improving any parametric model (Ho, Imai, King, \& Stuart, 2007). Matching procedures can overcome three problematic issues of regression analysis, the linearity assumptions, the need to extrapolate into unmeasured parts of the covariates, and capitalizing on unreliability when treating covariates singly (Cook, Steiner, \& Pohl, 2009). More details are provided in the Method section.

\section{Method}

The current investigation is part of the COGITO Study, conducted at the Center for Life span Psychology, Max Planck Institute for Human Development, Berlin (Lindenberger, Li, Lövdén, \& Schmiedek, 2007; Schmiedek, Bauer, Lövdén, Brose, \& Lindenberger, 2010). The COGITO Study followed a pretest-posttest control group design, with a microlongitudinal study phase of 100 days in the experimental group at its core. The present study reports data from this microlongitudinal phase.

\section{Participants and Procedure}

This study included 101 younger (51.5\% women, age: $20-31$ years, $M=25.6, S D=2.7)$ and 103 older participants $(49.5 \%$ women; age: $65-80$ years, $M=71.3, S D=4.1)$. Participants were recruited through newspaper advertisements, word-of-mouth recommendation, and flyers distributed in university buildings, community organizations, and local stores. The microlongitudinal phase of this study (daily sessions of 1-1.5 hours) was scheduled on an individual basis from Mondays to Saturdays between 8 a.m. and 7.30 p.m. The total number of sessions per person ranged from 87 to $107(M=101)$. During daily sessions, participants worked individually on cognitive tasks and answered questions on daily stressors, affect, motivation, and health in rooms with three to six work places. Incentives for study participation varied between 1450,- and 1950,- Euros. A bonus system was implemented to reduce attrition and increase motivation to complete the study quickly. For additional information on procedures, see Brose et al., 2011.

\section{Measures}

Daily negative affect. To assess negative affect, the 10-item negative affect subscale of the Positive and Negative Affect Schedule (PANAS; Watson, Clark, \& Tellegen, 1988) was used. Momentary affect was rated on an eight-point scale (0: does not apply at all, 7: applies very well). Five items (guilty, scared, hostile, ashamed, afraid) were excluded from analyses because they showed low variability across the study (20\% of the participants did not fluctuate at all on these items). The internal consistency of the remaining items (distressed, jittery, nervous, upset, and irritable) was calculated across subjects for a given session, resulting in an average Cronbach's alpha of .87 (ranging from .81 to .91 on Sessions 1 to 100). Individuals' average scores across the five items were used for analyses.

Daily stressors. A list of event categories was composed based on the Daily Inventory of Stressful Experiences
(Almeida, Wethington, \& Kessler, 2002) and a review of event questionnaires (Zautra, Affleck, \& Tennen, 1994). For each of seven events, participants were asked whether they had experienced the event since the last time they had come to the lab or whether such an event would occur later during that day: Having an argument with someone; having a disagreement over something without clarification; some event related to work; to a friend; to health; to leisure; and to finances. We also asked whether so many things requiring action or attention cooccurred that it could be called "stress" (overload category). If events had occurred, participants were asked to rate their valence (negative, slightly negative, neutral, slightly positive, or positive). Negative or slightly negative were counted as stressors. At the between-person level, we computed the number of days with at least one stressor, the total number of stressors, and chronic health-related stressors (for details, see below).

Daily rating of stressor impact on routines. As an additional context characteristic, the impact of stressors on daily routines was assessed with three items that asked for the extent to which (1) contact with other people was hindered by daily events, (2) routines were disturbed by daily events, and (3) participants were irritated because something unexpected had occurred during the last 24 hours. The last item was adopted from the Perceived Stress Scale (Cohen, Kamarck, \& Mermelstein, 1983). Again, an eight-point response scale from 0 (does not apply at all) to 7 (applies very well) was used. The average score across the three items was used in the analyses. Providing answers to the first two items requires an evaluation of context, which is more in line with this study's intentions than reporting one's irritation elicited by context. Given the high internal consistency of the three items (mean Cronbach's alpha $=.77$, ranging between .65 and .77 across the 100 sessions), we nevertheless decided to combine items into one score.

Operationalizing affect variability and affective reactivity. Consistent with our previous publication using the present data (Brose et al., 2011), affect variability was operationalized as the intraindividual standard deviation of individuals' affect ratings across study time, and affective reactivity was operationalized as the increase in negative affect on days with any of the stressors reported above as compared with days without stressors. Note that this is an imperfect (though widely used) index of affective reactivity. It does not reflect the temporal ordering between stressor occurrence and shifts in affect. Thus, we assume reactivity but cannot prove it.

\section{Analytical Procedure}

First, adult age group differences in stressor characteristics (stressors frequency, heterogeneity of stressors, stressor impact on daily routines) were determined. To do so, several aggregate scores were developed from the information that participants pro-

\footnotetext{
${ }^{2}$ Our adaptation of propensity score matching is at odds with various aspects of its common use. Context variables (the covariates used to calculate the propensity score) are not affecting selection into either age group. We adjust for what may actually be mediators; we only equate groups on context variables and not all variables. Our study is explicitly not on causality, and aging cannot be considered a treatment. Our reasoning behind the adaptation of PSM was thus not inspired by this method's typical aims, but in our view it is nonetheless well justified (see main text).
} 
vided on daily stressors (operationalized as daily events rated as negative or slightly negative, see above). These aggregate scores are presented in combination with the testing of the corresponding hypotheses in the Results section.

Second, we examined how stressor characteristics were related to emotional stability and age-group differences therein by means of correlation and multiple linear regression analysis. To examine affective reactivity, multiple linear regression was done in a multilevel modeling framework to account for the hierarchical structure of the data. The analyses were carried out in SAS PROC MIXED with Level-2 predictors centered on the sample mean. The autoregressive structure of the data was modeled with the SPATIAL POWER covariance function in the REPEATED statement, a procedure that takes differences in intervals between measurement occasions into consideration. Variance components corresponding to fixed effects were tested with likelihood ratio tests, using cutoff values provided by Stoel, Garre, Dolan, \& Van den Wittenboer (2006).

Third, we used a matching procedure that is in practical terms similar to PSM (Foster, 2010) to substantiate the findings from multiple regression analysis, given the criticism of this method as applied in our study (Cook et al., 2009; Ho et al., 2007). Propensity score matching was adapted following these steps: (1) We used logistic regression analysis to calculate propensity scores, that is, the likelihood of being in the younger or older group given all context characteristics. (2) Propensity scores were used to match samples (i.e., to obtain subsamples of younger and older adults with similar stressor characteristics). (3) We estimated the effect sizes of age-group differences in affect variability and affective reactivity in the matched samples and compared them with the effect sizes obtained in the total sample. Differences between both comparisons imply that age-group differences in affect variability and affective reactivity are related to variables characterizing context. Put differently, finding more similar affective variability and reactivity in subsamples of older and younger adults that were alike on context characteristics would be in line with our expectation that context is related to age-group differences in emotional stability.

Although matching as carried out in this study cannot overcome the limits of cross-sectional comparisons for establishing causal relationships between study variables (Lindenberger, von Oertzen, Ghisletta, \& Hertzog, 2011; Maxwell \& Cole, 2007), this analytical procedure has three important advantages over multiple regression: (1) Numerous variables characterizing daily life can be adjusted for simultaneously, which corresponds to the notion that affective experiences in different age groups occur in life contexts that differ in many regards; (2) age-related differences between matched and nonmatched samples can be compared without necessarily meeting the assumptions formulated in multiple regression analysis, in particular, on linearity among all variables (i.e., adjustment is separated from this analytical step); and (3) the matching procedure deleted the observations that would require substantial extrapolation (Cook et al., 2009; Ho et al., 2007). In multiple regression, the adjustment for covariates can lead to estimates of group differences that imply values on the covariates for which no observed cases actually exist in one or more of the groups to be compared. In the matched-sample analyses, the effect of age-group was examined after observations outside the region of overlap of younger and older adults on the covariates were excluded, thereby eliminating the necessity to extrapolate. This approach therefore tries to answer the question directly, "how DO younger and older adults differ in affect variability and reactivity if they HAVE comparable profiles of stressor contexts," rather than trying to get estimates for the question of, "how WOULD younger and older adults differ in affect variability and reactivity if they HAD comparable profiles of stressor contexts." Such an approach could fail if no region of overlap exists. In such cases, however, multiple regression particularly runs the risk of making inferences that are supported only under assumptions that are strong and untestable with the data at hand.

\section{Results}

\section{Age Differences in Stressor Characteristics}

First, we compared life contexts of younger and older adults. We expected that older in comparison with younger adults would experience fewer stressors, less heterogeneous stressors, stressors with less impact on routines, and more chronic stressors in the health domain.

To determine stressor frequency, we calculated two indices for each individual, the number of days with stressors across study time and the total number of stressors. When calculating the frequency measures, chronic stressors (i.e., stressors that occurred on more than $90 \%$ of the occasions) were excluded because chronic conditions are conceptually distinct from daily stressors. Both indices of stressor frequency yielded the expected age difference (see Table 1).

To determine how heterogeneous individuals' stressor profiles were, we computed Blau's Index of diversity (Blau, 1977) for each individual,

$$
\widetilde{D}=1-\sum_{i=1}^{S}\left(\frac{n_{i}}{N}\right)^{2}
$$

where $i$ is one out of $S$ stressor categories ( $S=8$ in our study), $n_{\mathrm{i}}$ is the number of stressors belonging to a stressor category $i$, and $N$ is the total number of stressors. According to this equation, stressor heterogeneity is largest if the number of stressors is equally distributed across categories. Stressor heterogeneity decreases the more stressors are concentrated around few categories. Theoretically, the index can have values ranging between 0 (no heterogeneity, i.e., all stressors fall in one category) and 1 (equal distribution of events over categories). For limited numbers of categories, the maximum value is less than 1 (e.g., .9 in the case of eight categories). Figure 1 illustrates how different individuals' stressor distributions are reflected in the index of heterogeneity. As expected, older adults' stressor profiles were less heterogeneous than younger adults' (see Table 1).

Table 1 also contains information on the average stressor impact on daily routines and on chronic health-related stressors. Confirming expectations, stressors were less disruptive on routines for older than for younger adults, and older adults reported more chronic health related stressors that may impose constraints on life contexts. 
Table 1

Age Group Differences in Central Study Variables

\begin{tabular}{|c|c|c|c|c|}
\hline & $\begin{array}{c}\text { Younger adults } \\
M(S D)\end{array}$ & $\begin{array}{l}\text { Older adults } \\
M(S D)\end{array}$ & $\begin{array}{l}\text { Age group } \\
\text { difference }\end{array}$ & Cohen's $d$ \\
\hline Number of days with stressors & $37(27)$ & $25(25)$ & $F(1,202)=10.36^{*}$ & 0.46 \\
\hline Number of stressors & $65(58)$ & $41(43)$ & $F(1,202)=11.31^{*}$ & 0.47 \\
\hline Heterogeneity of stressors ${ }^{\mathrm{a}}$ & $.72(.15)$ & $.59(.23)$ & $F(1,195)=11.25^{*}$ & 0.67 \\
\hline Stressor impact on routines & $1.62(1.20)$ & $0.62(0.81)$ & $F(1,202)=49.82^{*}$ & 0.98 \\
\hline Number of individuals with chronic health stressors & 2 & 15 & $\chi^{2}(3)=9.94^{*}$ & \\
\hline Affect variability & $0.76(0.34)$ & $0.34(0.28)$ & $F(1,202)=89.36^{*}$ & 1.36 \\
\hline Affective reactivity $^{\mathrm{b}}$ & $0.48(0.30)$ & $0.23(0.20)$ & $t(20333)=-5.22^{*}$ & .78 \\
\hline
\end{tabular}

${ }^{\mathrm{a}} n=197 .{ }^{\mathrm{b}}$ Fixed effect and random variation around fixed effect $(S D)$ generated with multilevel modeling.

${ }^{*} p=.05$

\section{Linking Stressor Characteristics to Affect Variability and Affective Reactivity}

Next, we examined the relationship between stressor characteristics and emotional stability, and whether age-group differences in stressor characteristics were related to previously reported agerelated reductions in affect variability and reactivity (Brose et al., 2011). In Table 2, we present correlations between all variables and partial correlations of stressor characteristics with affect variability and affective reactivity, adjusting for age group. Results mainly followed expectations: Interindividual differences in affect variability were positively related to interindividual differences in days with stressors, number of stressors, heterogeneity of stressor profiles, and the impact of stressors on routines. Likewise, interindividual differences in stressor heterogeneity and the impact of stressors on routines were related to interindividual differences in affective reactivity, although the frequency indices were unrelated to affective reactivity. Chronic health-related stressors were not correlated with affect variability and affective reactivity (both $p$ values >.75). Therefore, this variable is not reported in Table 2 and will not be included in the following analyses.

Adjusting for age group in the correlations between affect variability, affective reactivity, and context variables and then comparing the correlation and partial correlation coefficients reveals first evidence for shared variance among age, context characteristics, and emotional stability. In particular, when ad-

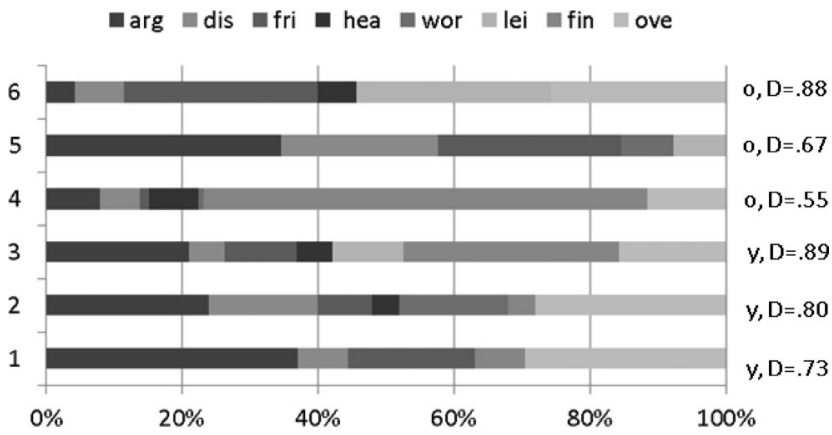

Figure 1. Illustration of heterogeneity of participants' stressor profiles: three younger (y) and three older (o) adults, selected according to rank order of diversity index (D), rank 30, 60, 90; stressor types: $\arg =$ argument; dis $=$ disagreement; fri $=$ friends; hea $=$ health; wor $=$ work; lei $=$ leisure; fin $=$ finances; ove $=$ overload. justing for age group in the correlations between affect variability and context variables, the partial correlations with stressor frequency, stressor heterogeneity, and the impact of stressors on routines is reduced. A similar pattern emerged for affective reactivity.

Affect variability. To examine the extent to which agegroup differences in affect variability can be predicted by interindividual differences in context variables, three successive regression models were tested. In each, the amount of explained variance was determined. First, age was included as a predictor of affect variability. Second, the four variables representing context were included simultaneously as predictors of variability. Third, age and the four variables were included simultaneously. Thereby we were able to distinguish between those portions of the total explained variance that were shared by age and context variables and those that were unique to age and context (see Figure 2). Age group uniquely explained $9 \%$ of the total variance in affect variability, context uniquely explained $21 \%$, and the shared predictive variance between age and context was $21 \%$. Thus, reduced affective variability in older adults was associated with context characteristics (the frequency of stressors, heterogeneity of stressor occurrences, and the impact of stressors on routines).

Affective reactivity. Next, we turn to age group differences in affective reactivity. The model equation used in these analyses was

Level-1 NA $_{i j}=\beta_{0 i}+\beta_{1 i}\left(\right.$ Session $\left._{i j}\right)+\beta_{2 i}\left(\right.$ Daily Stressors $\left._{i j}\right)+r_{i j}$

Level-2 $\beta_{0 i}=\gamma_{00}+\gamma_{01}\left(\right.$ Age Group $\left._{i}\right)+\gamma_{02}\left(\right.$ Stressors Days $\left._{i}\right)$

$+\gamma_{03}\left(\right.$ Number Stressors $\left._{i}\right)+\gamma_{04}\left(\right.$ Heterogeneity $\left._{i}\right)$

$+\gamma_{05}\left(\right.$ Stressor Impact on Routines $\left._{i}\right)+\mathrm{u}_{0 i}$

$$
\begin{aligned}
\beta_{1 i}= & \gamma_{10}+\mathrm{u}_{1 i} \\
\beta_{2 i}= & \gamma_{20}+\gamma_{21}\left(\text { Age Group }_{i}\right)+\gamma_{22}\left(\text { Stressors Days }_{i}\right) \\
& +\gamma_{23}\left(\text { Number Stressors }_{i}\right)+\gamma_{24}\left(\text { Heterogeneity }_{i}\right) \\
& +\gamma_{25}\left(\text { Stressor Impact on Routines }_{i}\right)+\mathrm{u}_{2 i}
\end{aligned}
$$

In the Level-1 equation, negative affect of person $i$ on occasion $j$ is predicted by the person-level intercept, $\beta_{0 i}$, a linear slope to adjust for mean trends, $\beta_{1 i}$, and the occurrence of a stressor on day 
Table 2

Correlations (Lower Triangle, Bold Numbers) and Partial Correlations (Adjusting for Age Group; Upper Triangle) Between Study Variables

\begin{tabular}{|c|c|c|c|c|c|c|c|}
\hline & Age group & Var & React & Days stressors & Number stressors & Het & Stressor impact \\
\hline Affect variability (var) & $-0.55^{*}$ & & $0.67^{*}$ & $0.30^{*}$ & $0.39^{*}$ & $0.27^{*}$ & $0.48^{*}$ \\
\hline Affective reactivity (react) & $-0.37^{*}$ & $0.72^{*}$ & & -0.08 & 0.05 & $0.17^{*}$ & $0.21^{*}$ \\
\hline Days with stressors & $-0.22^{*}$ & $0.35^{*}$ & -0.01 & & $0.92^{*}$ & 0.003 & $0.47^{*}$ \\
\hline Number of stressors & $-0.23^{*}$ & $0.43^{*}$ & 0.12 & $0.92^{*}$ & & $0.11^{*}$ & $0.51^{*}$ \\
\hline Stressor heterogeneity (het) & $-0.26^{*}$ & $0.36^{*}$ & $0.25^{*}$ & 0.06 & $0.16^{*}$ & & $0.20^{*}$ \\
\hline Stressors impact on routines & $-0.45^{*}$ & $0.64^{*}$ & $0.34^{*}$ & $0.51^{*}$ & $0.55^{*}$ & $0.28^{*}$ & \\
\hline
\end{tabular}

Note. Partial correlations (adjusting for age group) point to shared variance between age group and both of the remaining two variables (e.g., a reduction in the correlation between stressor heterogeneity and affect variability when adjusting for age group indicates that the association between age and affect variability is not independent from interindividual differences in stressor heterogeneity); the intraindividual reactivity coefficients (react) used to calculate these correlations are the individual estimates derived from the random effect that was estimated together with the fixed effect in multilevel modeling; all but one of these individual estimates were positive.

${ }^{*} p<.05$.

$j, \beta_{2 i}$. In the level-2 equations, the intercept $\beta_{0 i}$ is predicted by the sample-level intercept, $\gamma_{00}$, the person's age group, $\gamma_{01}$, the number of days with stressors, $\gamma_{02}$, the number of stressors, $\gamma_{03}$, stressor heterogeneity, $\gamma_{04}$, and stressor impact on routines, $\gamma_{05}$. The same Level-2 variables predict $\beta_{2 i}$, the slope representing affective reactivity $\left(\gamma_{21}, \gamma_{22}, \gamma_{23}, \gamma_{24}, \gamma_{25}\right)$. Age group was coded as 0 (younger adults) and 1 (older adults). Person $i$ 's deviation from the samples' intercept is denoted by $u_{0 i}$, and $r_{i j}$ denotes person i's deviation from a predicted score at occasion $j$. The slope parameters, $\beta_{1 i}$ and $\beta_{2 i}$ were allowed to vary across individuals by including the random effects $u_{1 i}$ and $u_{2 i}{ }^{3}$

Again, we were interested in unique and shared predictive variance between age group and stressor characteristics, here, predicting affective reactivity. We followed the logic described above (successive models were fitted and the amount of variance in affective reactivity that was uniquely explained by age group, uniquely explained by context characteristics, and shared between these two was determined). Note that in contrast to the analysis involving affect variability, affective reactivity is an outcome variable at Level 2 in this multilevel model, $\beta_{2 i}$, that was predicted by variables at Level 2 (age group and context variables). The explained variance in reactivity was determined by comparing pseudo- $R^{2}$ statistics (Singer \& Willett, 2003).
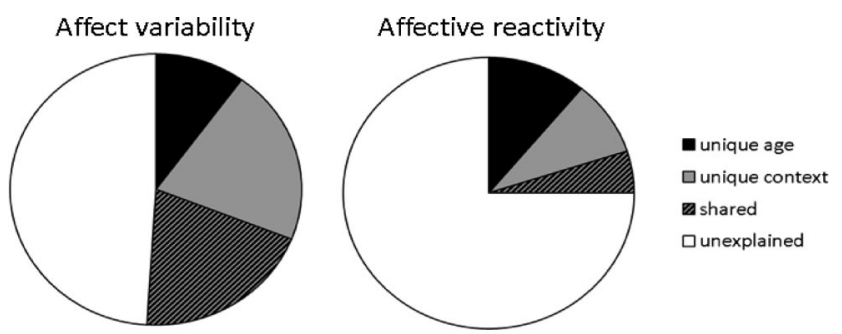

Figure 2. Results from multiple regression analyses: Variance explained in affect variability and affective reactivity; context variables are number of days with stressors (c1), number of stressors (c2), stressor heterogeneity (c3), and the impact of stressors on routines (c4); unique effects on affect variability in bivariate regressions (explained variance): age $=31 \% ; \mathrm{c} 1=$ $12 \% ; \mathrm{c} 2=18 \% ; \mathrm{c} 3=10 \% ; \mathrm{c} 4=37 \%$; unique effects on affect reactivity in bivariate multilevel-regressions (explained variance): age $=16 \%$; $1=$ $0 \% ; \mathrm{c} 2=0 \% ; \mathrm{c} 3=8 \% ; \mathrm{c} 4=6 \%$.
When affective reactivity was predicted by age group, $16 \%$ of variance was explained, and $15 \%$ of variance in affective reactivity was explained by context variables. Age group and context variables jointly explained $24 \%$ of variance in affective reactivity. Thus, age group uniquely explained $9 \%$ of the total variance in affective reactivity, context uniquely explained $8 \%$ of the total variance in affective reactivity, and the shared predictive variance was $7 \%$ (see Figure 2). Thus, reduced affective reactivity in older adults was associated with characteristics of daily life, but relatively larger parts of age and context variables predicted affective reactivity independently.

\section{Affect Variability and Reactivity in Samples Matched on Stressor Characteristics}

We proceeded with matching younger and older adults on context variables to examine affect variability and affective reactivity in subgroups that were similar in stressor characteristics. We expected affect variability and affective reactivity to be more similar in these matched subsamples than in total sample.

The propensity of being in the younger or older group given all context information was estimated by means of logistic regression (predictors: days with stressors, number of stressors, stressor heterogeneity, impact of stressors on routines). The distributions of the estimated propensity scores in the two age groups are presented in Figure 3. The average propensity score significantly differed between the younger and older adults of total sample, $F(1,202)=$ $60.46, p<.0001$. Before logistic regression, we replaced missing values in the heterogeneity index with 0 to keep all individuals for matching (missing values occurred in two younger and seven older

${ }^{3}$ The fixed effect for Session (on affect) was significant in this model, $\beta_{1 i}=-0.001, t=2.36, p=.02 ; \Delta-2 L L=944$, d.f. $=2, p<$ .05 (an increase by 0.1 scale point units across 100 occasions, and this change differed interindividually). A quadratic change component was not significant. We also tested for linear decrease in the predictor (stressors) because of a potential reactivity to measurement procedures (i.e., decrease in motivation). Stressor reports decreased across study time, $\beta_{1 i}=-1.1791, t=10.36, p<.05$. The likelihood to report events decreased from 29 to $24 \%$, and there were interindividual differences therein. This change in reports had a negligible effect on the coupling coefficient, $r_{\text {individual_slopes_uncorrected, individual_slopes_corrected }}=.98$. Therefore, the uncorrected predictor was kept. 


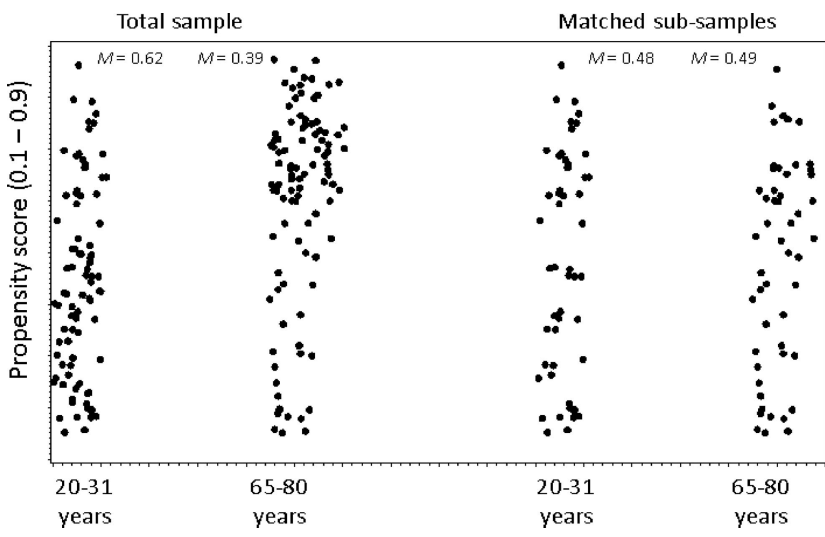

Figure 3. Distribution of propensity scores in the total sample and in matched subsamples of younger and older adults; each dot represents one individual; the individuals in the matched samples originate from the total sample.

adults who did not report any stressors). This way, all individuals could be kept for matching.

Next, subsamples of younger and older adults were matched on the basis of this propensity score using nearest neighbor matching in the SAS macro program MATCH. Matching was done within the common range of scores across age groups. Of 204 individuals, 102 subjects could be matched to 51 pairs $\left(n_{\text {younger }}=51, n_{\text {older }}=\right.$ 51 ). These did no longer differ on the propensity score, $F(1,100)=$ 0 , ns, nor on any of the variables characterizing context (all $p$ values $>.05$; all effect sizes of mean differences across age groups $<0.02$ ). Comparing the selected subsamples with the remaining younger and older adults revealed that the selected younger adults did not differ from their unselected peers in terms of gender and age, $F(1,99)=0.01, p>.05$ and $F(1,99)=2.42$, $p>.05$, respectively. The selected older adults did not differ from their unselected peers in terms of gender, $F(1,101)=1.64, p>$ .05 , but they did differ in terms of age, $F(1,101)=4.28, p<.05$ $\left(M_{\text {selected }}=70.5, M_{\text {unselected }}=72.1\right)$.

Finally, we compared the means of affect variability and affective reactivity in the matched subsamples and calculated the effect sizes of the age-group differences. Individuals' variability and reactivity scores were the same as reported above (the intraindividual standard deviation across study time and individuals' reactivity coefficients obtained from the mixed model with all subjects). These effect sizes were then compared with the effect sizes of age-group differences in the total sample. Results are presented in Figure 4. Despite the fact that the selected groups of younger and older adults were comparable on stressor characteristics, affect variability in the matched sample was still smaller in older than in younger adults, $F(1,100)=18.3, p<.05$. Important, however, was that the effect size in the matched samples was significantly reduced to 0.86 as compared with 1.36 in the total sample $(95 \%$ confidence intervals of the effect sizes did not include the respective other effect size). Affective reactivity also remained smaller in older than younger adults in the matched samples, $F(1,100)=$ 13.13. The effect size was reduced to 0.71 in comparison with 0.78 in the total sample. In this case, both $95 \%$ CIs of the effect sizes included the respective other effect size. Thus, the effect size in the matched samples was not significantly smaller than in the total sample.

These findings provide further evidence that age-group differences in emotional stability are related to age-differential life contexts, albeit only partially. The age-group difference in affect variability - though not in affective reactivity — was significantly reduced in the subsamples. That is, given more similar environments, younger and older adults' variability was more alike. At the same time, the age-group differences in affect variability and affective reactivity remained significant, indicating that life contexts as operationalized in this study can only partly explain older adults' increased emotional stability.

These results were stable when the propensity score was estimated with only the frequency and heterogeneity indices as predictors. We did this follow-up analysis because stressor impact on routines may partly reflect regulatory skills (i.e., the ability to regulate stressors such that they do not impact on routines). Seventy matched pairs were identified in this follow-up analysis. The effect size of the age-group difference in affect variability was $d=$ .96 in this subsample $\left(95 \% \mathrm{CI}: 0.61-1.31 ; M_{\text {younger }}=0.70\right.$ $\left.M_{\text {older }}=0.40\right)$. The effect size of the difference in affect reactivity was $d=.64$ in this subsample $\left(95 \% \mathrm{CI}: 0.30-0.98 ; M_{\text {younger }}=\right.$ $\left.0.46, M_{\text {older }}=0.28\right)$. Thus, the age-group difference in affect

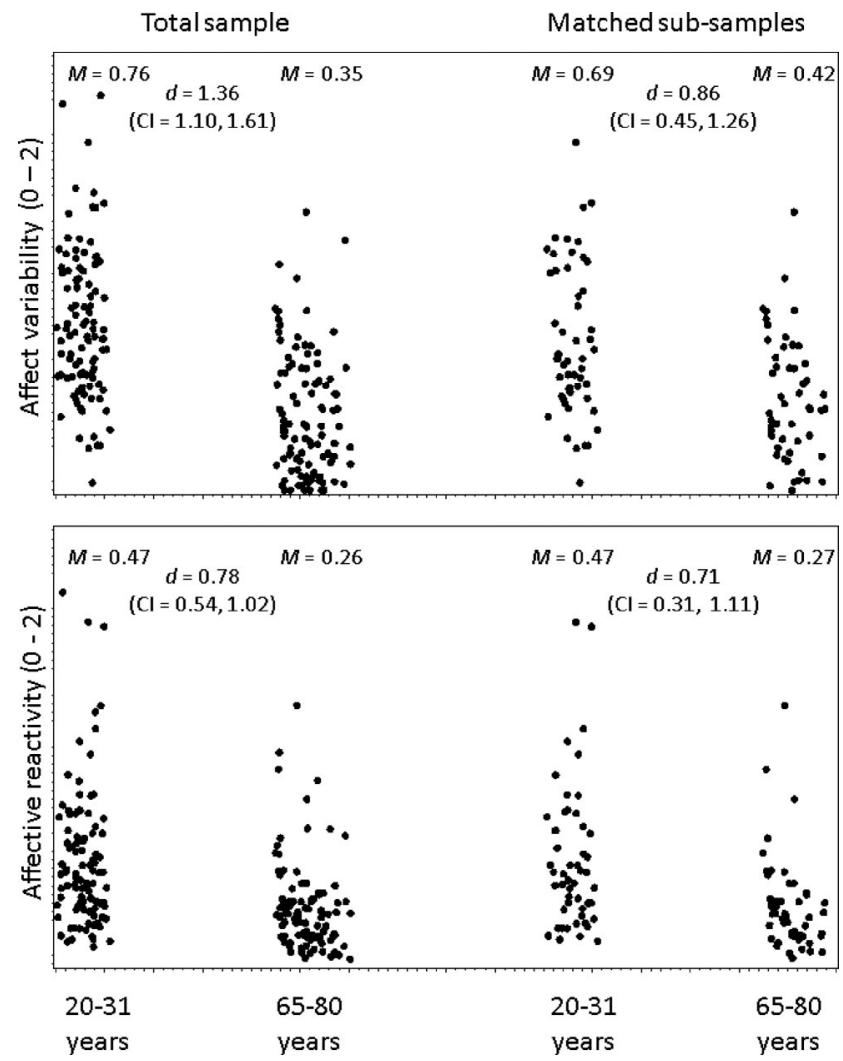

Figure 4. Results from the matching procedure: Affect variability and affective reactivity in the total sample and in matched samples; dots represent values from single individuals; the individuals in the matched samples originate from the total sample; means refer to the average affect variability and average affective reactivity in the total sample and in the matched subsamples. 
variability was again significantly smaller in this subsample than in the total sample (for the effect size and CI for comparison, please see Figure 4). The age-group difference in affective reactivity again did not differ from the one that was observed in the total sample.

\section{Discussion}

Emotional aging has various facets, causes, and consequences. Our study allows a closer look into the context in which daily life, and thus, daily affective experiences take place, based on selfreports of stressful events across 100 days. We established links between life contexts and emotional stability as well as life contexts and age-related differences in emotional stability. Our findings support the ideas that interindividual differences in emotional stability may partly occur because of differences in life contexts and that age differences in emotional stability may partly occur because of normative age-group differences in life contexts. Thus, the findings of this study are supportive of a partially contextual explanation of adult age differences in emotional functioning. At the same time, age-group differences in emotional stability were not eliminated when adjusting for life contexts, which may imply that the development of affective experiences is driven by both, changes in the environment and in the person.

Specifically, in this extensive 100-day long investigation of daily lives, our hypotheses were supported that older adults experience fewer stressors, that the heterogeneity of domains in which stressors occur is reduced, that older adults report stressors that are less disruptive of daily routines than younger adults', and that older adults experience more chronic health-related stressors. Also in line with our expectations, relatively low affect variability and little affective reactivity occurred in individuals with more homogeneous stressor profiles and with stressors that were less disruptive of daily routines. That is, interindividual differences in emotional stability are related to context. Heterogeneity in emotional well-being between individuals and in trajectories of emotional well-being may therefore partly be explainable by contextual variables. The newly developed stressor heterogeneity index proved to be an important situational moderator of affect variability and stress reactivity that should be considered in future research on stress, distress, and well-being in addition to situational determinants such as stressor severity or stressor type (Almeida et al., 2011). Finally, we showed that significant proportions of the age-related variance in affect variability were associated with between-person differences in life contexts. Subgroups of younger and older adults who were relatively alike in life context were also more alike in affect variability. Results generated with multiple regression analysis point in the same direction for affective reactivity. Yet, as we believe the matching procedure is the superior method for answering our research question (see below), we conclude that context makes a difference for variability, but potentially not for reactivity. This finding is particularly noteworthy in light of the conceptual and empirical overlap between variability and reactivity. Longitudinal follow-up studies as well as statistical analyses that incorporate time are required before statements about change and causality are possible (Maxwell \& Cole, 2007).

The findings of this study qualify the literature on emotional aging in important ways. Much of the more recent debate on adult age differences in emotional functioning has focused on mecha- nisms that reside within the individual. The literature has focused on changes in motivation and the ability to regulate emotions and on expertise based on prior exposure (Blanchard-Fields et al., 2007; Carstensen et al., 1999; Charles, 2010; Scheibe \& Carstensen, 2010). However, the context in which any behavior occurs and develops has always been a crucial aspect in developmental theory (Baltes, Lindenberger, \& Staudinger, 2006; Bronfenbrenner \& Ceci, 1994). Its role for emotional functioning was debated in earlier writings on habituation (Kastenbaum, 1981), and it is present in cross-cultural research on aging (Fung, Stoeber, Yeung, \& Lang, 2008). Yet, context effects are remarkably neglected in most of the current discourse on emotional aging.

The findings of this study strongly suggest that life contexts should receive more attention when attempting to understand interindividual and age-group differences in emotional functioning. When the unique and shared predictive variance of age and contextual variables (i.e., number of days with stressors, the total number of stressors, stressor heterogeneity, stressors' impact on daily routines) are disentangled, about $40 \%$ of the explained variance in affect variability was shared by age and context characteristics. Regarding the explained variance in affective reactivity, about $29 \%$ was identified as shared predictive variance. Moreover, results generated with the matching procedure further support context effects on affect variability. Interestingly, the age-group difference in context characteristics may be particularly relevant for age-group differences in affect variability and less so for those in affective reactivity. A potential reason is that when a threshold is passed for an event to be appraised as stressful, coping, including emotion regulation, likely is elicited. In such contexts of specific stressful events, potential age-related improvements in emotion regulation may come into play and contain affective reactivity. In other words, age-related differences in emotion regulation may become more relevant when facing stressors than in the ebb and flow of more mild and subtle influences on affect. Thus, the relative contribution of psychological moderators seems to be higher for affective reactivity than for variability, which is in line with a functional distinction between affect variability and reactivity

Potentially, effects of life contexts on emotional stability may be even more pronounced than what can be inferred from this study. Although we took several contextual characteristics into consideration, those are but a few from a much broader range of contextual differences in younger and older adults' lives. Adjusting for more contextual characteristics may attenuate age-group differences in variability even further, and this may also attenuate age-group differences in reactivity. For example, in future studies, researchers may want to count the number of distinguishable contexts, of social interactions, or of alternations between locations, potentially by using ecological momentary assessment (Mehl \& Connor, 2012)

Although this highlights the importance of context for agegroup specific emotional experiences, an interactionist perspective according to which behavior is a function of both the person and the situation (Steyer, Schmitt, \& Eid, 1999) is probably more correct than an either-or position. Age-group differences in life contexts that were identified may in part result from regulation efforts. Notwithstanding normative age-graded differences, individuals are agents and shape their environment (Brandtstädter \& Lerner, 1999). Reporting about the environment means to some 
extent constructing it, and fewer events could be construed as distressing by older adults (Charles et al., 2009). Two potential reporting biases are positive reappraisal or positively biased memory, both of which have been linked to age. Such biases are thought to serve regulatory functions, which lowers the clear distinction between the context and psychological processes of mastering context. In the related vein, the emotion regulation strategies of situation selection and situation modification may partly account for age-group differences in life contexts (e.g., older adults seem to structure their social world such that negative interactions are avoided; Carstensen et al., 2003; Urry \& Gross, 2010). Only future studies can reveal the extent to which objective versus subjective aspects of changing contexts are related to emotional functioning, the extent to which older adults shape their environment, and whether they do so to regulate emotions and to achieve any overarching goal of well-being.

\section{Strengths and Limitations}

An obvious strength of this study is the large number of occasions, which allowed the measurement of context by including both frequent and infrequent stressors, and also allowed the determination of stressor heterogeneity per individual. A second particular strength of this study is its focus on matching rather than regression analysis. Admittedly, results of the matching procedure as applied in this study are no closer to causality and longitudinal change than the results generated with regression analysis in cross-sectional data sets (for criticisms of the use of cross-sectional data for generating insights on change and mediation, see Lindenberger et al., 2011; Maxwell \& Cole, 2007). Nevertheless, matching procedures are advantageous in comparison with multiple regression analysis as detailed in the Introduction and Method sections (numerous contextual variables were adjusted for simultaneously and before testing the effect of age on emotional stability; no linearity assumptions on the relationship between covariates and the outcome variable were needed; it was not necessary to extrapolate, which would rely on the assumption that the same relationships exist between variables across subgroups and for those expressions of variables that are outside a range common across subgroups - an assumption that is often criticized as unrealistic). In sum, matching as applied in this study appears to be a promising alternative to multiple regression because there are fewer problematic technical aspects in situations in which one is interested in age-related differences while adjusting for (numerous) covariates.

It has to be kept in mind that the subsample of older adults that was matched to a subsample of younger adults with comparable stressor profiles was younger than the nonmatched subsample of older adults (70.5 vs. 72.1 year on average). This is in line with our reasoning, however, that beyond midlife, increasing age is associated with less stressful contexts. It thus seems plausible to find more matches for younger adults in the "young-old" participants. As in multiple regression approaches to adjust for confounding covariates, it remains unknown whether the matched participants are selected on other variables that could account for this study's finding of more similar emotional stability in subsamples of younger and older adults with comparably stressful life contexts. Based on theoretical considerations, such additional variables could be included in the matching procedure.
This study has an additional obvious limitation. Insights on life contexts were gained with self-report. Various distortions may apply. If habituation to stressors occurs across life span (Frederick \& Loewenstein, 1999), the threshold beyond which stressors are reported as stressors might be higher in older adults. Relatedly, if the immediate affective reaction to a stressor is minor in older adults (e.g., Brose et al., 2011), some events perceived as stressors by younger adults may not be perceived as such by older adults. More generally, even if different individuals report the same stressors, this does not mean that the stressors are alike in intensity. Vice versa, if individuals report different stressors, the same events may nevertheless have occurred. However, objective measurement of daily events also entails difficulties because, by definition, an event is stressful when it is perceived as such. A second disadvantage of our proxy of context is its potential overlap with the affect measure. Event evaluations (e.g., whether an event is perceived as routine-disrupting) may depend on current affect. Again, confounds could only be prevented by objective measurements. In sum, there are difficulties with our proxy of the environment, but an ideal solution to this issue is difficult to achieve.

Future studies would benefit from including middle-aged adults. For them, contextual demands may be particularly high because of high responsibilities in the work and family domain. Assuming that context drives emotional stability, this would suggest a curvilinear age trajectory of emotional stability (but see Carstensen et al., 2011, who showed a linear increase in affect variability). Using a matching procedure as we suggest would be particularly wellsuited to investigate the relationship between context and emotional stability across the whole period of adulthood if the trajectory of contextual demands were nonlinear.

\section{Conclusion}

Life contexts differ within and also across age groups. Results of this study suggest systematic age differences in an important aspect of life contexts, namely stressor profiles. In comparison with younger adults, older adults' lives were less stressful in the sense that they reported fewer stressors, that stressors were less heterogeneous, and that stressors had less impact on routines. Importantly, life context differences were related to dynamic aspects of emotional well-being, namely affect variability and reactivity, in both age groups. We found support for the assumption that older adults' reduced affect variability is associated with age-specific contexts. Results were less conclusive for affective reactivity. We conclude that context factors require more attention in future studies on the development of emotional well-being. It remains to be disentangled to which extent context is an antecedent or a consequence of regulatory efforts; that is, to which extent the context per se or rather the individual shaping the context determine emotional outcomes.

\section{References}

Almeida, D. M., \& Horn, M. C. (2004). Is daily life more stressful during middle adulthood? In O. G. Brim, C. D. Ryff \& R. C. Kessler (Eds.), How healthy are we? A national study of well-being at midlife (pp. 425-451). Chicago, IL: The University of Chicago Press.

Almeida, D. M., Stawski, R. S., \& Cichy, K. E. (2010). Combining checklist and interview approaches for assessing daily stressors: The 
Daily Inventory of Stressful Experiences. In R. J. Contrada \& A. Baum (Eds.), The handbook of stress science: Biology, psychology, and health. New York, NY: Springer.

Almeida, D. M., Wethington, E., \& Kessler, R. C. (2002). The Daily Inventory of Stressful Experiences: An interview-based approach for measuring daily stressors. Assessment, 9, 41-55.

Baltes, P. B., Lindenberger, U., \& Staudinger, U. M. (2006). Lifespan theory in developmental psychology. In W. Damon \& R. M. Lerner (Eds.), Handbook of child psychology: Vol. 1. Theoretical models of human development (pp. 569-664). New York, NY: Wiley.

Blanchard-Fields, F., Mienaltowski, A., \& Seay, R. B. (2007). Age differences in everyday problem-solving effectiveness: Older adults select more effective strategies for interpersonal problems. The Journals of Gerontology: Series B: Psychological Sciences and Social Sciences, 62, P61-P64. doi:10.1093/geronb/62.1.P61

Blau, P. M. (1977). Inequality and heterogeneity. New York, NY: Free Press.

Bolger, N., \& Zuckerman, A. (1995). A framework for studying personality in the stress process. Journal of Personality and Social Psychology, 69, 890-902. doi:10.1037/0022-3514.69.5.890

Bouisson, J. (2002). Routinization preferences, anxiety, and depression in an elderly French sample. Journal of Aging Studies, 16, 295-302. doi:10.1016/S0890-4065(02)00051-8

Brandtstädter, J., \& Lerner, R. M. (1999). Action and self-development. Theory and research through the life span. Thousand Oaks, CA: Sage.

Bronfenbrenner, U., \& Ceci, S. J. (1994). Nature-nurture reconceptualized in developmental perspective: A bioecological model. Psychological Review, 101, 568-586. doi:10.1037/0033-295X.101.4.568

Brose, A., Schmiedek, F., Lövdén, M., \& Lindenberger, U. (2011). Normal aging dampens the link between intrusive thoughts and negative affect in reaction to daily stressors. Psychology and Aging, 26, 488-502. doi: 10.1037/a0022287

Carstensen, L. L., Isaacowitz, D. M., \& Charles, S. T. (1999). Taking time seriously: A theory of socioemotional selectivity. American Psychologist, 54, 165-181. doi:10.1037/0003-066X.54.3.165

Carstensen, L. L., Mayr, U., Pasupathi, M., \& Nesselroade, J. R. (2000). Emotional experience in everyday life across the adult life span. Journal of Personality and Social Psychology, 79, 644-655. doi:10.1037/00223514.79.4.644

Carstensen, L. L., Turan, B., Scheibe, S., Ram, N., Ersner-Hershfield, H., Samanez-Larkin, G. R., . . . Nesselroade, J. R. (2011). Emotional experience improves with age: Evidence based on over 10 years of experience sampling. Psychology and Aging, 26, 21-33. doi:10.1037/ a0021285

Carver, C. S. \& Scheier, M. F. (1999). Themes and issues in the selfregulation of behavior. In R. S. Wyer, Jr. (Ed.), Perspectives on behavioral self-regulation: Advances in social cognition, Vol. XII. (pp. 1-105). Mahwah, NJ: Lawrence Erlbaum Associates.

Charles, S. T. (2010). Strength and vulnerability integration: A model of emotional well-being across adulthood. Psychological Bulletin, 136, 1068-1091. doi:10.1037/a0021232

Charles, S. T., Luong, G., Almeida, D. M., Ryff, C., Sturm, M., \& Love, G. (2010). Fewer ups and downs: Daily stressors mediate age differences in negative affect. The Journals of Gerontology: Series B: Psychological Sciences and Social Sciences, 65, 279-286. doi:10.1093/geronb/gbq002

Cohen, S., Kamarck, T., \& Mermelstein, R. (1983). A global measure of perceived stress. Journal of Health and Social Behavior, 24, 385-396.

Cook, T. D., Steiner, P. M., \& Pohl, S. (2009). Assessing how bias reduction is influenced by covariate choice, unreliability and data analytic mode: An analysis of different kinds of within-study comparisons in different substantive domains. Multivariate Behavioral Research, 44, $828-847$.

Dohrenwend, B. P. (2006). Inventorying stressful life events as risk factors for psychopathology: Toward resolution of the problem of intracategory variability. Psychological Bulletin, 132, 477-495. doi:10.1037/00332909.132.3.477

Eid, M., \& Diener, E. (1999). Intraindividual variability in affect: Reliability, validity, and personality correlates. Journal of Personality and Social Psychology, 76, 662-676. doi:10.1037/0022-3514.76.4.662

Erikson, E. H. (1968). Identity: Youth and crisis. New York, NY: Norton

Eysenck, H. J. (1990). Genetic and environmental contributions to individual-differences - the 3 major dimensions of personality. Journal of Personality, 58, 245-261. doi:10.1111/j.1467-6494.1990.tb00915.x

Fleeson, W., \& Jolley, S. (2006). A proposed theory of the adult development of intraindividual variability in trait-manifesting behavior. In D. K. Mroczek \& T. D. Little (Eds.), Handbook of personality development (pp. 41-59). Mahwah, NJ: Erlbaum.

Foster, E. M. (2010). Causal inference and developmental psychology. Developmental Psychology, 46, 1454-1480. doi:10.1037/a0020204

Frederick, S., \& Loewenstein, G. (1999). Hedonic adaptation. In D. Kahneman, E. Diener \& N. Schwarz (Eds.), Well-being: The foundations of hedonic psychology (pp. 302-329). New York, NY: Russell Sage Foundation.

Freund, A. M., Nikitin, J., \& Ritter, J. O. (2009). Psychological consequences of longevity the increasing importance of self-regulation in old age. Human Development, 52, 1-37. doi:10.1159/000189213

Fung, H. H., Stoeber, F. S., Yeung, D. Y.-I., \& Lang, F. R. (2008). Cultural specificity of socioemotional selectivity: Age differences in social network composition among Germans and Hong Kong Chinese. The Journals of Gerontology: Series B: Psychological Sciences and Social Sciences, 63B, P156-P164. doi:10.1093/geronb/63.3.P156

Gross, J. J. (1998). Antecedent- and response-focused emotion regulation: Divergent consequences for experience, expression, and physiology. Journal of Personality and Social Psychology, 74, 224-237. doi: 10.1037/0022-3514.74.1.224

Guralnik, J. M., Lacroix, A. Z., Abbott, R. D., Berkman, L. F., Satterfield, S., Evans, D. A., \& Wallace, R. B. (1993). Maintaining mobility in late-life. 1. Demographic characteristics and chronic conditions. American Journal of Epidemiology, 13, 845-857.

Havighurst, R. J. (1973). Social roles, work, leisure, and education. In C. Eisdorfer \& M. P. Lawton (Eds.), The psychology of adult development and aging (pp. 598-618). Washington, DC: American Psychological Association. doi:10.1037/10044-019

Ho, D. E., Imai, K., King, G., \& Stuart, E. A. (2007). Matching as nonparametric preprocessing for reducing model dependence in parametric causal inference. Political Analysis, 15, 199-236. doi:10.1093/ $\mathrm{pan} / \mathrm{mp} 1013$

John, O. P., \& Gross, J. J. (2004). Healthy and unhealthy emotion regulation: Personality processes, individual differences, and life span development. Journal of Personality, 72, 1301-1334. doi:10.1111/j.14676494.2004.00298.x

Kahn, R. L., \& Antonucci, T. C. (1980). Convoys over the life course Attachment, roles, and social support. In P. B. Baltes \& O. Brim (Eds.), Life-span development and behavior (Vol. 3).New York, NY: Academic Press.

Kastenbaum, R. J. (1980-1981). Habituation as a model of human aging. The International Journal of Aging \& Human Development, 12, 159170. doi:10.2190/BR5F-H8B7-2B9X-53U8

Koole, S. (2009). The psychology of emotion regulation: An integrative review. Cognition and Emotion, 23, 4-41. doi:10.1080/ 02699930802619031

Kuppens, P., Oravecz, Z., \& Tuerlinckx, F. (2010). Feelings change: Accounting for individual differences in the temporal dynamics of affect. Journal of Personality and Social Psychology, 99, 1042-1060. doi:10.1037/a0020962

Lawton, M. P., Kleban, M. H., Rajagopal, D., \& Dean, J. (1992). Dimensions of affective experience in three age groups. Psychology and Aging, 7, 171-184. doi:10.1037/0882-7974.7.2.171 
Lazarus, R. S., \& Folkman, S. (1984). Stress, appraisal, and coping. New York, NY: Springer.

Lindenberger, U., Li, S. C., Lövdén, M., \& Schmiedek, F. (2007). The Center for Lifespan Psychology at the Max Planck Institute for Human Development: Overview of conceptual agenda and illustration of research activities. International Journal of Psychology, 42, 229-242. doi:10.1080/00207590701396591

Lindenberger, U., von Oertzen, T., Ghisletta, P., \& Hertzog, C. (2011). Cross-sectional age variance extraction: What's change got to do with it? Psychology and Aging, 26, 34-47. doi:10.1037/a0020525

Luhmann, M., \& Eid, M. (2009). Does it really feel the same? Changes in life satisfaction following repeated life events. Journal of Personality and Social Psychology, 97, 363-381. doi:10.1037/a0015809

Maxwell, S. E., \& Cole, D. A. (2007). Bias in cross-sectional analyses of longitudinal mediation. Psychological Methods, 12, 23-44. doi:10.1037/ 1082-989X.12.1.23

Mehl, M., \& Connor, T. (2012). Handbook of research methods for studying daily life. New York, NY: Guilford.

Mroczek, D. K., \& Almeida, D. M. (2004). The effect of daily stress, personality, and age on daily negative affect. Journal of Personality, 72, 355-378. doi:10.1111/j.0022-3506.2004.00265.x

Nesselroade, J. R. (1991). The warp and the woof of the developmental fabric. In R. M. Downs, L. S. Liben \& D. S. Palermo (Eds.), Visions of aesthetics, the environment and development: The legacy of Joachim F. Wohlwill (pp. 213-240). Hillsdale, NJ: Lawrence Erlbaum Associates.

Oswald, F., \& Wahl, H.-W. (2005). Dimensions of the meaning of home. In G. D. Rowles \& H. Chaudhury (Eds.), Home and Identity in Late Life: International Perspectives (pp. 21-45). New York, NY: Springer.

Ram, N., \& Gerstorf, D. (2009). Time-structured and net intraindividual variability: Tools for examining the development of dynamic characteristics and processes. Psychology and Aging, 24, 778-791. doi:10.1037/ a0017915

Riediger, M., \& Freund, A. M. (2006). Focusing and restricting: Two aspects of motivational selectivity in adulthood. Psychology and Aging, 21, 173-185. doi:10.1037/0882-7974.21.1.173

Robinson, M. D., \& Clore, G. L. (2002). Belief and feeling: Evidence for an accessibility model of emotional self-report. Psychological Bulletin, 128, 934-960. doi:10.1037/0033-2909.128.6.934

Röcke, C., Li, S. C., \& Smith, J. (2009). Intraindividual variability in positive and negative affect over 45 days: Do older adults fluctuate less than young adults? Psychology and Aging, 24, 863-878. doi:10.1037/ a0016276

Rosenkoetter, M. M., Garris, J. M., \& Engdahl, R. A. (2001). Postretirement use of time: Implications for preretirement planning and postretirement management. Activities, Adaptation \& Aging, 25, 1-18. doi: 10.1300/J016v25n03_01

Scheibe, S., \& Carstensen, L. L. (2010). Emotional Aging: Recent Findings and Future Trends. The Journals of Gerontology: Series B: Psychological Sciences and Social Sciences, 65, 135-144. doi:10.1093/geronb/ gbp 132

Schmiedek, F., Bauer, C., Lövdén, M., Brose, A., \& Lindenberger, U. (2010). Cognitive enrichment in old age. GeroPsych: The Journal of Gerontopsychology and Geriatric Psychiatry, 23, 59-67.

Seery, M. D. (2011). Resilience: A Silver Lining to Experiencing Adverse Life Events? Current Directions in Psychological Science, 20, 390-394. doi: $10.1177 / 0963721411424740$

Serido, J., Almeida, D. M., \& Wethington, E. (2004). Chronic stressors and daily hassles: Unique and interactive relationships with psychological distress. Journal of Health and Social Behavior, 45, 17-33. doi:10.1177/ 002214650404500102

Shiota, M. N., \& Levenson, R. W. (2009). Effects of aging on experimentally instructed detached reappraisal, positive reappraisal, and emotional behavior suppression. Psychology and Aging, 24, 890-900. doi:10.1037/ a0017896

Singer, J. D., \& Willett, J. B. (2003). Applied longitudinal data analysis: Modeling change and event occurrence. New York, NY: Oxford University Press.

Sliwinski, M. J., Almeida, D. M., Smyth, J., \& Stawski, R. S. (2009). Intraindividual change and variability in daily stress processes: Findings from two measurement-burst diary studies. Psychology and Aging, 24, 828-840. doi:10.1037/a0017925

Stawski, R. S., Almeida, D. M., Lachman, M. E., Tun, P. A., \& Rosnick, C. B. (2010). Fluid cognitive ability is associated with greater exposure and smaller reactions to daily stressors. Psychology and Aging, 25, 330-342. doi:10.1037/a0018246

Stawski, R. S., Sliwinski, M. J., Almeida, D. M., \& Smyth, J. M. (2008). Reported exposure and emotional reactivity to daily stressors: The roles of adult age and global perceived stress. Psychology and Aging, 23, 52-61. doi:10.1037/0882-7974.23.1.52

Steyer, R., Schmitt, M., \& Eid, M. (1999). Latent state-trait theory and research in personality and individual differences. European Journal of Personality, 13, 389-408. doi:10.1002/(SICI)1099-0984(199909/10)13: 5<389::AID-PER361>3.0.CO;2-A

Stoel, R. D., Garre, F. G., Dolan, C., \& Van den Wittenboer, G. (2006). On the likelihood ratio test in structural equation modeling when parameters are subject to boundary constraints. Psychological Methods, 11, 439455. doi:10.1037/1082-989X.11.4.439

Uchino, B. N., Berg, C. A., Smith, T. W., Pearce, G., \& Skinner, M. (2006). Age-related differences in ambulatory blood pressure during daily stress: Evidence for greater blood pressure reactivity with age. Psychology and Aging, 21, 231-239. doi:10.1037/0882-7974.21.2.231

Urry, H. L., \& Gross, J. J. (2010). Emotion Regulation in Older Age. Current Directions in Psychological Science, 19, 352-357. doi:10.1177/ 0963721410388395

van Eck, M., Nicolson, N. A., \& Berkhof, J. (1998). Effects of stressful daily events on mood states: Relationship to global perceived stress. Journal of Personality and Social Psychology, 75, 1572-1585. doi: 10.1037/0022-3514.75.6.1572

Watson, D., Clark, L. A., \& Tellegen, A. (1988). Development and validation of brief measures of positive and negative affect: The PANAS scales. Journal of Personality and Social Psychology, 54, 1063-1070. doi:10.1037/0022-3514.54.6.1063

Williams, D. G. (1990). Effects of pychoticism, extraversion, and neuroticism in current mood - a statistical review of 6 studies. Personality and Individual Differences, 11, 615-630. doi:10.1016/01918869(90)90045-S

Wolff, J. K. (2007). Stimmungen und Stimmungsschwankungen: Einflüsse von Alter und Persönlichkeit [Mood and mood variability: Relationships with age and personality]. Unpublished diploma thesis, FriedrichSchiller-Universität, Jena, Germany.

Wrzus, C., Müller, V., Wagner, G. G., Lindenberger, U., \& Riediger, M. (2012). Affective and cardiovascular responding to unpleasant events from adolescence to old age: Complexity of events matters. Developmental Psychology. Advance online publication. doi:10.1037/a0028325

Zautra, A. J., Affleck, G., \& Tennen, G. (1994). Assessing life events among older adults. In M. P. Lawton \& J. A. Teresi (Eds.), Annual review of gerontology and geriatrics: Focus on assessment techniques (pp. 324-352). New York, NY: Springer.

Zautra, A. J., Finch, J. F., Reich, J. W., \& Guarnaccia, C. A. (1991). Predicting the everyday life events of older adults. Journal of Personality, 59, 507-538. doi:10.1111/j.1467-6494.1991.tb00258.x

Received March 23, 2012

Revision received July 12, 2012

Accepted August 1, 2012 